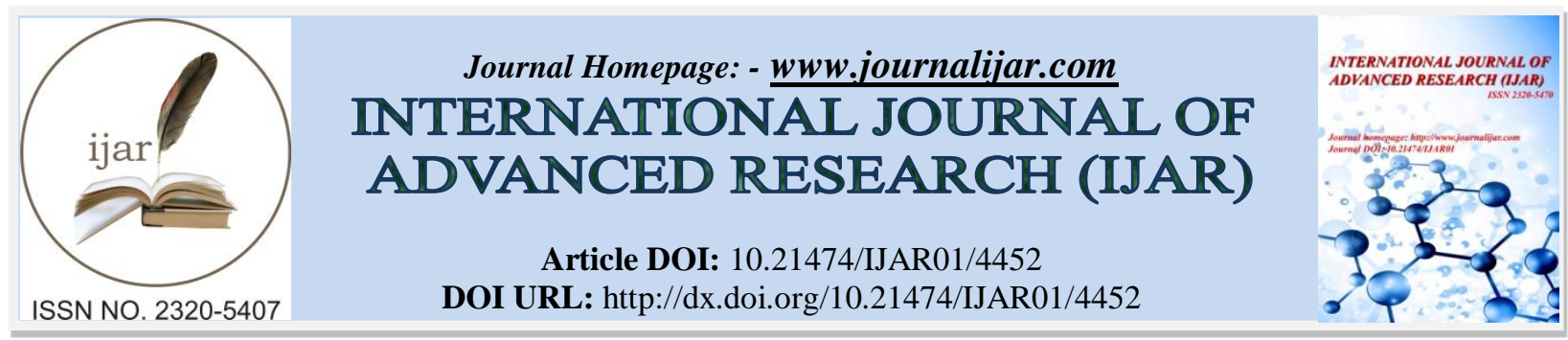

RESEARCH ARTICLE

\title{
IMPACT OF METFORMIN AND ITS DURATION OF USE ON SEVERITY OF ISCHEMIC STROKE IN PATIENTS WITH TYPE 2 DIABETES MELLITUS.
}

\author{
Alamelu $\mathrm{AL}^{1}$ and Dhananjayan Kannan ${ }^{2}$. \\ 1. $3^{\text {rd }}$ year MBBS, Govt. Kilpauk Medical College and Hospital. \\ 2. Senior Assistant Professor of Medicine, Govt. Kilpauk Medical College and Hospital.
}

\section{Manuscript Info}

(n........................

Manuscript History

Received: 11 April 2017

Final Accepted: 13 May 2017

Published: June 2017

Key words:-

Ischemic stroke; Diabetes mellitus; metformin; severity of stroke; NIHSS scale; mRS scale.

\section{Abstract}

Background: Stroke is one of the most devastating neurological conditions. Diabetes mellitus is a well-established independent risk factor for stroke. Metformin therapy reportedly decreases the risk of stroke. But the association between the duration of metformin therapy and the severity of stroke has not yet been assessed. This study aims at assessing the impact of metformin and its duration of use on the severity of ischemic stroke in patients with type $2 \mathrm{DM}$.

Methods: We assessed 60 stroke patients with type 2 DM, without severe renal impairment (serum creatinine $<1.7 \mathrm{mg} / \mathrm{dl}$ ) who were admitted to Kilpauk medical college hospital between July 2016 and September 2016. Severity of stroke was assessed using NIHSS scale and $\mathrm{mRS}$ scale.

Results: Metformin therapy was associated with decreased severity of stroke showing improvements in functional outcomes. $(p=0.017, \mathrm{CI}-$ $95 \%$ ). In contrast, no significant correlation was found between the duration of metformin use and severity of stroke.

Conclusion: Pretreatment with metformin therapy in patients with type 2 diabetes mellitus reduces the severity of stroke. However the correlation between the duration of metformin use and severity of stroke was not significant.

Ischemic stroke; Diabetes mellitus; metformin; severity of stroke; NIHSS scale; mRS scale.

Copy Right, IJAR, 2017,. All rights reserved.

\section{Introduction:-}

Stroke is a non-communicable disease, which remains one of the most devastating of all neurological conditions. In 2010, an estimated 16.9 million cases of incident stroke took place worldwide with 33 million prevalent stroke cases, 5.9 million stroke death and 102.2 million DALYs lost(1).The estimated prevalence rate of stroke in India is $84-262 / 100,000$ in rural and 334-424/100,000 in urban areas(2).

The major risk factors for stroke as identified by The Framingham Heart Study and other international prospective epidemiological studies are hypertension, diabetes mellitus, hyperlipidemia and smoking (3).

Diabetes mellitus is a well-established independent risk factor for stroke and is associated with high mortality. The Atherosclerosis Risk in Community Study (ARIC) showed that the risk attributable to diabetes was approximately $21 \%$. 
Metformin has become a mainstay in the modest therapeutic armamentarium for the treatment of insulin resistance of type 2 diabetes mellitus. It is on the World Health Organization's List of Essential Medicines, the most important medications needed in a basic health system. Nearly $80 \%$ of patients with type 2 diabetes mellitus are under metformin supplements.

Gundewar et al(4) quoted that, metformin in addition to anti-hyperglycaemic effects, provides additional cardioprotective effects related to lipid metabolism, vascular smooth muscle and cardiomyocyte calcium handling, endothelial function, coagulation and platelet activity.

Increasing epidemiological evidence suggests that metformin reduces stroke incidence and severity. These results indicate that metformin could be developed into a disease modifying drug to treat stroke(5).

This study assessed the impact of metformin and its duration of use on severity of ischemic stroke using NIHSS score and mRS scale in patients with type 2 Diabetes mellitus.

\section{Methods:-}

A total of 60 stroke patients with type $2 \mathrm{DM}$, without severe renal impairment (serum creatinine $<1.7 \mathrm{mg} / \mathrm{dl}$ ) who were admitted to Kilpauk medical college hospital between July 2016 and September 2016 were assessed.

\section{Data collection and clinical assessment:-}

Information regarding the patient's medical history, vital signs, stroke type, NIHSS score, mRS score and laboratory findings were collected. Blood investigations for plasma glucose and serum creatinine were done. The stroke type was classified according to the classification of cerebrovascular disease as proposed by the National Institute of Neurological Disorders and Stroke. The diagnosis of Diabetes mellitus was made based on the criteria of the American Diabetes Association. Renal function was assessed by the serum creatinine level. The severity of stroke was assessed by the National Institute of Health Stroke Scale (NIHSS) score and the functional outcomes by modified Rankin Scale (mRS) score. Mild neurological severity is defined as an NIHSS score less than 3 on admission.

The NIHSS and mRS scoring assessment were done by primary investigator. All biochemical analyses were done with adequate internal quality checks and within run and between run CV (coefficient variations) were maintained. Informed consent were obtained from all the patients.

\section{Statistical Analysis:-}

Coded data was entered in excel sheet and analysed with SPSS 20 package. The severity of stroke in both groups were expressed as percentage with $95 \%$ confidence interval. The correlation between stroke severity and metformin was expressed as correlation coefficient (spearman). $\mathrm{t}$ - test was used for testing significance between proportions. $\mathrm{p}$ $<0.05$ was considered as statistically significant

\section{Results:-}

The purpose of this study was to find the impact of metformin and its duration of use on the severity of ischemic stroke in patients with type 2 diabetes mellitus. Hence a comparative cross sectional study which includes 30 ischaemic stroke patients with past history of type 2 diabetes who are under metformin supplements and 30 ischaemic stroke patients with past history of type 2 diabetes who are not under metformin supplements. This study was conducted in the Department of Internal Medicine and Biochemistry in our institution.

The exclusion criteria includes patients with severe renal impairment, having serum creatinine greater than 1.7 $\mathrm{mg} / \mathrm{dl}$, since metformin is contraindicated in these patients (6).

The mean age of case is 59.033 , the mean age of control is 54.833 with $\mathrm{p}$ value of $0.98(\mathrm{p}$ value $>0.05)$. The cases and controls were age matched.

Males (cases - 53.33\%, control - 83.33\%) were more affected by ischemic stroke than females (cases - $46.67 \%$, control - 16.67\%), which is similar to the study done by Peter Appelros et al (7). It might be due to the beneficial effects of estrogen on cerebral circulation. 
No significant difference in fasting plasma glucose levels ( $\mathrm{p}$ value -0.903 . $\mathrm{p}$ value $>0.05$ ) were seen between cases (mean - 196.9) and controls (mean - 200.867).

The mean serum creatinine level of cases is 1.217 , the mean serum creatinine level of control is 1.167 , with p value of 0.628 ( $\mathrm{p}$ value $>0.05$ ). The cases and controls were matched for serum creatinine level.

The NIHSS score is statistically not significant with $\mathrm{p}$ value of 0.114 ( $\mathrm{p}$ value $>0.05$ ). But the NIHSS score is higher in controls (mean - 11.933) than in cases (mean - 8.867), which is similar to the results observed by Yohei mima et al (8).

The mRS score is statistically significant with $\mathrm{p}$ value of 0.017 ( $\mathrm{p}$ value $<0.05$ ). The $\mathrm{mRS}$ score is greater in controls (mean - 3.867) than in cases (mean - 3.3). This shows that metformin users have favourable functional outcomes when compared to metformin nonusers.

From the ROC curve, the sensitivity and specifity of NIHSS score is $73.3 \%$ and $63.3 \%$ respectively with the cut off value of 7.5. The sensitivity and specificity of mRS scale is $80 \%$ and $46.7 \%$ respectively with the cut off value of 3.50 .

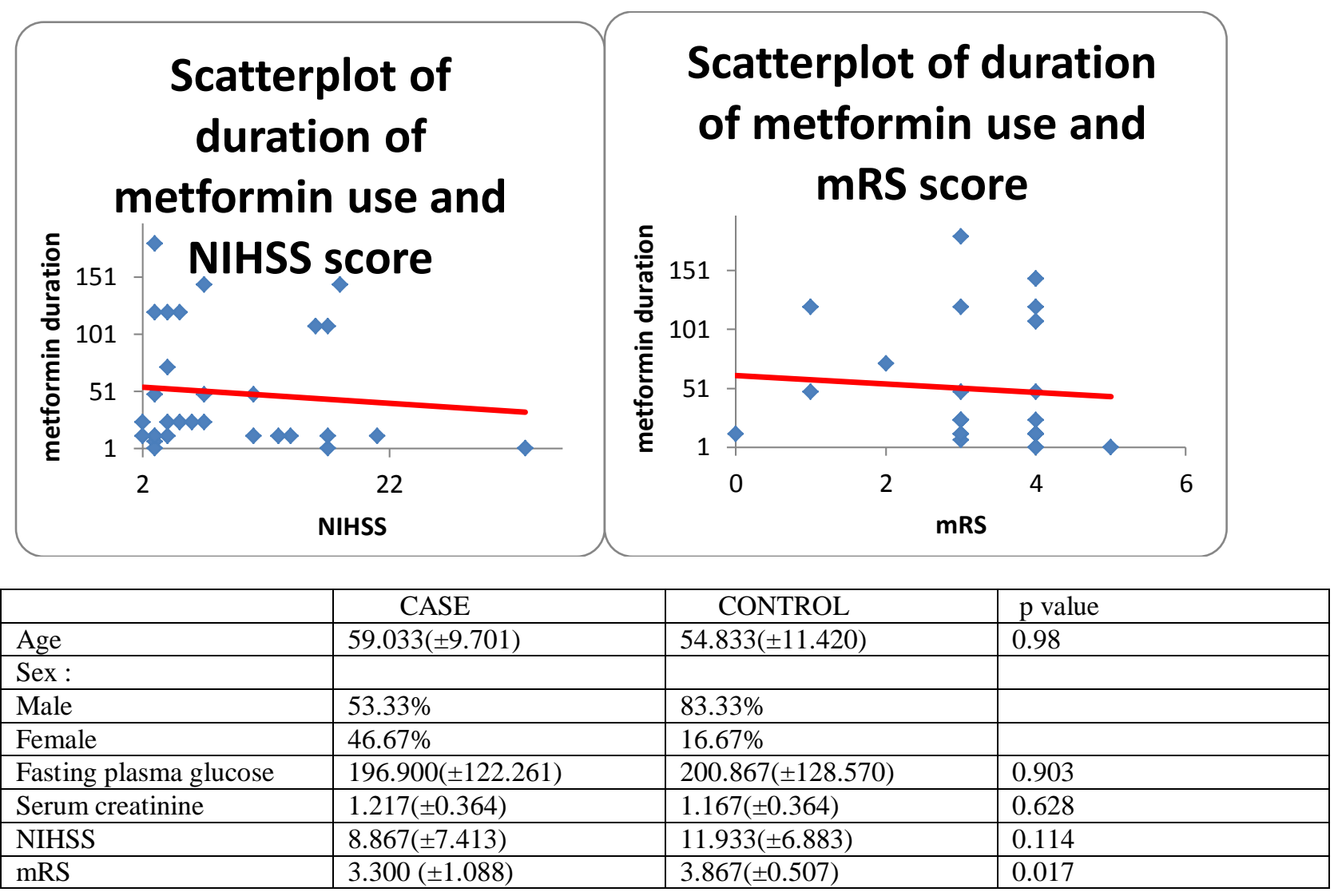

\section{Discussion:-}

In this study, the mRS score is statistically significant indicating that metformin users have favourable function outcomes. This is due to the fact that metformin activates AMPK in hepatocytes, as a result, acetyl coA carboxylase activity is reduced, fatty acid oxidation is induced and expression of lipogenic enzymes are suppressed (9).

In addition to the anti-hyperglycemic effects, metformin provides additional cardioprotective effects beyond sugar lowering, which may be related to the actions of metformin on lipid metabolism, vascular smooth muscle and cardiomyocyte intracellular calcium handling, endothelial function, hyper-coagulation and platelet hyperactivity. It 
also provides greater protection against the development of macrovascular complications than would be expected from its effects on glycemic control alone.

In previous studies, metformin treatment significantly improved glycation, oxidative stress, nitric oxide (NO) bioavailability and insulin resistance and normalized endothelial function in vessels of rats with diabetes. In human studies, persons who received metformin had improvement in endothelium-dependent, acetylcholine-stimulated flows compared with those treated with placebo.

It has been reported that metformin treatment was associated with improvements in plasma markers of endothelial function, including von Willebrand factor (vWF), soluble vascular adhesion molecule-1 (sVCAM-1), tissue-type plasminogen activator (t-PA), plasminogen activator inhibitor-1 (PAI-1) and soluble intercellular adhesion molecule-1 (sICAM-1), suggesting the benefit of endothelial function improvement and inflammation reduction(10).

Several other studies indicate that day long plasma concentration of TG, RLP-C and sVCAM-1 were lower after metformin therapy (11).

Long term metformin therapy shows a significant reduction in total cholesterol concentration, reduction in LDL cholesterol without any change in HDL cholesterol, high significant reduction in PAI-1 levels with no significant effect on fibrinogen levels. Thus long term metformin treatment could reduce the risk of thrombosis through its effect on fibrinolysis by lowering PAI-1 concentrations (12).

The improvements in the cerebrovascular outcomes seen with metformin is due to the decrease in PAI-1 that accompanies metformin induced increase in insulin sensitivity.

These findings indicate that metformin is able to improve endothelial reactivity at the macro- and microcirculatory level, both of which relate to better cerebrovascular outcomes.

Thus, metformin may have better effect on endothelial protection and coagulation, which may provide possible mechanisms connected to the reduced risk for cerebrovascular accidents.

In this study, there is no significant correlation between duration of metformin use and both NIHSS score and mRS score.

Jun Li et al(13) suggested that acute metformin usage (less than 3 days) exacerbated stroke damage whereas chronic use of metformin (greater than 3 weeks) was neuroprotective. This implies that metformin can exacerbate or ameliorate stroke severity which is entirely dependent on its duration of use.

Chirantan et al (14), stated that the duration of diabetes is independently associated with ischemic stroke risk.

The duration of metformin usage in this study was elicited from the treatment history of patients. A proper documentation and recording of treatment history and duration of diabetes, with a greater sample size would give promising results.

\section{Conclusion:-}

Both NIHSS and mRS scores were lower in cases when compared to controls, however the correlation between the NIHSS score, mRS score and the duration of metformin use was not significant.

\section{Summary:-}

This comparative cross sectional study was conducted with 30 ischaemic stroke patients under metformin supplements and 30 ischaemic stroke patients not under metformin supplements to assess the impact of metformin and its duration of usage on the severity of stroke in patients with type 2 diabetes mellitus. The severity of stroke is lower in cases when compared to controls, but the correlation between the duration of metformin use and the severity of stroke was not significant. 


\section{Further scope for improvement:-}

1. A larger population size could increase the statistical significance of study.

2. Proper documentation and recording of treatment history of the patients and duration of diabetes would give promising results.

\section{References:-}

1. Valery L Feigin, Mohammad H Forouzanfar, Rita Krishnamurthi. Global and regional burden of stroke during 1990-2010: findings from the Global Burden of Disease Study 2010. Lancet 2014; 383: 245-55.

2. Jeyaraj Durai Pandian and Paulin Sudhan. Stroke Epidemiology and Stroke Care Services in India. J Stroke. 2013 Sep; 15(3): 128-134.

3. Tapas Kumar banerjee, Shyamal Kumar. Epidemiology of stroke in India. Neurology Asia 2006; $11: 1-4$.

4. S Gundewar et al. Activation of AMPK Metformin improves left ventricular function and survival in heart failure. Circ Res,2009 Feb 13; 104(3): 403-411.

5. Jia J, et al. Neuropharmacological Actions of Metformin in Stroke. Curr Neuropharmacol. 2015.13,389-394.

6. Kasia J. lipska, Clifford J. Bailey, Silivo E. inzucchi. Use of metformin in the setting of mild to moderate renal insufficiency. Diabetes care 2011 June; 34(6): 1431-1437.

7. Peter Appelros, Birgitta Stegmayr, Andreas Terent. Sex differences in stroke epidemiology. Stroke. 2009;40: 1082-1090.

8. Yohei mima, Takahiro kuwashiro, Masahiro yasaka. Impact of metformin on severity and outcomes of acute ischaemic stroke in patients with type 2 diabetes mellitus. Journal of stroke and cerebrovascular diseases. Feb 2016, vol.25(2):436-446.

9. Gaochao Zhou, Robert Myers, Ying Li. Role of AMP-activated protein kinase in mechanism of metformin action. J Clin Invest. 2001;108(8): 1167-1174.

10. Dai Yin $\mathrm{Lu}$, et al. Metformin use in patients with type 2 diabetes mellitus is associated with reduced risk of deep vein thrombosis: a non-randomized, pair-matched cohort study. BMC Cardiovasc Disord. 2014 14:187.

11. Fahim Abbasi, James W. Chu, Tracey McLaughlin. .Effect of Metformin Treatment on Multiple Cardiovascular Disease Risk Factors in Patients With Type 2 Diabetes Mellitus. Metabolism, Vol 53, No 2 (February), 2004: pp 159-164.

12. UK Prospective Diabetes Study (UKPDS) Group. Effect of intensive blood-glucose control with metformin on complications in overweight patients with type 2 diabetes. The Lancet 12 September 1998, Vol.352(9131):854865

13. Jun Li, Sharon E. benashski, Venugopal Reddy Venna. Effects of metformin in experimental stroke. Stroke. 2010 Nov: 41(11): 2645-2652.

14. Chiratan Banerjee, Yeseon P Moon, Mitchell SV Elkind. Duration of diabetes and risk of ischemic stroke: the northern Manhattan study. Stroke. 2012 May; 43(5): 1212-1217. 